\title{
EFFECT OF YOGA ON CARDIOVASCULARAUTONOMIC REACTIVITY IN ESSENTIAL HYPERTENSIVE PATIENTS
}

\author{
Khadka $\mathrm{R}^{1}$, Paudel $\mathrm{BH}^{1}$, Sharma $\mathrm{VP}^{2}$, Kumar $\mathrm{S}^{1}$, Bhattacharya $\mathrm{N}^{1}$ \\ ${ }^{1}$ Department of Physiology, ${ }^{2}$ Department of Medicine \\ BPKIHS, Dharan, Nepal
}

\begin{abstract}
Background and objective: We aimed to investigate the effect of yoga on cardiovascular autonomic reactivity in essential hypertensive patients. Methods: The study was conducted on 14 essential hypertensive patients, who were on salt-reduction and similar antihypertensive drugs. They were randomized into two groups; control $(n=7$; age $42.2 \pm 11.9$ years) and yoga $(n=7$; age $44.9 \pm 10.8$ years). The yoga group practiced yoga for $1 / 2 \mathrm{~h} / \mathrm{d}, 6 \mathrm{~d} /$ week for 6 weeks. The control group did not practice any type of yogic exercises or relaxation techniques. Autonomic function tests consisting of deep breathing, Valsalva maneuver, handgrip (HGT), and head-up tilt (HUT) tests of all patients were assessed at 0 week and every two weeks for 6 weeks. The data were analyzed using Friedman test followed by multiple comparisons. Relusts: In yoga group significant reduction in resting heart rate, SBP, and DBP were found after 4 weeks of yogic practices as compared to baseline recording; HR [84(78-94.5) Vs 72(67.5-77); $\mathrm{p}=0.028]$, SBP[144(140-165) Vs 130(125144); $\mathrm{p}=0.018]$, and DBP[98(94-101) Vs 88(78-90); $\mathrm{p}=0.018]$. Similarly, significant reduction in SBP was found after yogic practices in response to HGT and HUT although the magnitude of changes was increased or comparable to control. There was significant increase in Valsalva ratio after yogic practices [1.17(1.12-1.4) Vs 1.33(1.29-1.55); $\mathrm{p}=0.018]$. Conculsion: Yoga combined with anti-hypertensive drugs is effective in reducing BP in resting condition and increasing parasympathetic reactivity. It is also found to normalize cardiovascular autonomic function in hypertensive patients.
\end{abstract}

Keywords: Yoga, essential hypertension.

\section{Introduction}

Hypertension is one of the most prevalent cardiovascular disorders affecting $20 \%$ of world's population. ${ }^{1}$ Eighteen percent of it is essential hypertension. The central venous pressure as well as arterial blood pressure is increased in essential hypertensive patients. The elevated central venous pressure is due to decrease in compliance of either the venous bed of left ventricle or both; and it is amplified with age and baseline vascular resistance. ${ }^{2}$ Sympathetic nervous hyper-tonicity may be

Address for correspondence:

Dr Rita Khadka

Assistant Professor, Department of Physiology, BPKIHS

Email: rita.khadka@gmail.com responsible for activation of the renin-angiotensin system, and both of these together may play an important role in the elevation of BP. ${ }^{3}$ Release of nor-epinephrine from central nervous system (CNS), presumably in the forebrain, mediates increased sympathetic nerve firing in patients with essential hypertension. ${ }^{4}$ Studies on heart rate variability have shown decreased parasympathetic activity in essential hypertensive patients. ${ }^{5,6}$

Stress has been implicated as one of the major causes of essential hypertension by producing large amount of vasoconstrictor hormones that increase BP. ${ }^{7}$ Persistent increase in arterial BP may result in cardiovascular (ventricular hypertrophy, myocardial infarction, and stroke) and other complications. 
As the exact causes of the disease is yet to be known and the nature of the disease is multi-factorial, many patients have to take anti-hypertensive drugs lifelong for the management of the essential hypertension. Long-term treatment and multiple drug requirements make the patients' compliance poor. Despite long-term normalization of BP by anti-hypertensive drugs, there exists autonomic dysfunction. ${ }^{8}$ Therefore, there is a continuous search for newer alternative methods of therapeutics.

Based on limited scientific research, yoga (meditation, asanas, and pranayama) including relaxation therapy is known to improve cardiovascular autonomic functions. Meditation is associated with reduced sympathetic adrenergic receptor sensitivity, which might affect cardiovascular response during stress. ${ }^{9}$ During meditation appearance of frontal midline theta rhythm in electroencephalogram reflects mental concentration as well as meditative state of relief from anxiety and is correlated negatively with sympathetic activation. This suggests a close relationship between autonomic functions and activity of medial frontal neural circuitry and possibility of controlling CNS functions through yoga and meditation. ${ }^{10}$ Transcendental meditation (TM) practice improves mood state, adrenocortical activity and kidney functions; and believed to reduce stress ${ }^{11}$ and shows significant reduction in average ambulatory diastolic BP. ${ }^{12}$ Its effect is additive in reducing $\mathrm{BP}$ in patients treated with $\mathrm{a}$-blockers and $\mathrm{Ca}^{++}$channel blockers.

The yogic asanas influence various organs physiologically, exert neuro-physiological stability by lowering level of cholinesterase and catecholamines, and show certain significant trends of endocrine restoration and metabolic correction. ${ }^{13}$ The Shawasana-a relaxation exercise probably influence the hypothalamus- can establish a psychophysiological relaxation in a short period. Therefore, we aimed to investigate the effect of yoga (meditation, pranayama and certain easy asanas) on cardiovascular autonomic responses in essential hypertensive patients.

\section{Material and methods}

The study was conducted on 14 ambulatory essential hypertensive patients, age ranging from $30-60$ years of either sex. They were newly diagnosed and follow-up cases, previously not engaged in any type of relaxation therapy. Patients with concomitant diseases (diabetes mellitus, secondary and severe hypertension) were excluded from the study. All the recruited patients were taking â-blocker as well as $\mathrm{Ca}^{++}$channel blockers and were on salt reduction. Patients were systematically randomized into control and yoga groups. The control group was on conventional drug therapy and salt reduced diet. The yoga group was on similar anti- hypertensive drugs and salt reduction, and also practiced certain selected yogic practices for half an hour per day, 6 days in a week for 6 weeks. They practiced yoga under the supervision of trained yoga instructor at Yoga and Life Style Clinic, Department of Physiology, BPKIHS. The study was conducted from 20002002.

The yoga group practiced the following yogic practices

1. Strenthening exercise (for $\mathbf{5}$ min): consisted of Manibadha, Shakti Vikash, Ardhabhuja Shakti Vikash, Purna Shakti Vikash, Anjuli Shakti Viksh, Kamar Chakrasana,Vakshasthal Shakti Vikash and Uder Shakti Vikash. Each exercise was done for $40 \mathrm{sec}$.

2. Yogic asanas (for $6-\mathbf{- 7} \mathbf{~ m i n}$ ): consisted of Tarasana, Trikonasana, Gomudhasana, Shashankasana, Padmasana, Bhujangasana, Hardhayastambhasana, Naukasana and Makarasana. Each was done for $40 \mathrm{sec}$.

3. Shavasana, a relaxation exercise, was done for 5 min.

4. Meditation was done in a comfortable posture (desired by patients) for $5 \mathrm{~min}$.

5. Pranayama: consisted of Anuloma-biloma and Nadisuddi pranayama. Both are breathing exercises. Each was done for $2 \mathrm{~min}$.

The assessment of autonomic function test (AFT) was performed in both the groups before randomization and at every two weeks for 6 weeks between 8:30 to 11:00 AM at room temperature $26 \pm 1{ }^{\circ} \mathrm{C}$ after 2 hours of breakfast. The AFT consisted of deep breathing test, Valsalva maneuver, handgrip test (HGT) and head-up tilt test (HUT). ${ }^{14}$ During all these tests simultaneous ECG and respiration were recorded continuously. Deep breathing, Valsalva maneuver and HGT were done as recommended by Ewing (1982). ${ }^{15}$ For head-up tilt test patients were asked to lie down supine on a tilt table for $15 \mathrm{~min}$ and then table was tilted at $60^{\circ}$ in $15 \mathrm{sec}$ and the patients were remained tilted for 6 
min. Baseline BP was recorded in supine position at the end of supine rest and subsequent BP was recorded at $1 / 2 \mathrm{~min}, 1 \mathrm{~min}, 2 \mathrm{~min}, 4 \mathrm{~min}$, and $6 \mathrm{~min}$ in the tilted position. After 6 min patients were brought to the horizontal position.

\section{Statistical analysis}

The data were tested for normal distribution. The data of general characteristics was analyzed using unpaired student $t$ test. The Friedman test followed by multiple comparisons was used to study the changes within the group over the period of time and Mann Whitney $U$ test was used for inter group comparison. A p value of $<0.05$ was considered statistically significant.

\section{Results}

There were no significant differences between yoga and control groups in terms of their age, sex, height, weight, and blood pressure (Table 1). The yoga group showed significant reduction in weight and body mass index (BMI) after four weeks of yoga practice as compared to baseline recording and control group (Table 2). The yoga group also showed significant reduction in resting HR, SBP, and DBP after 4 weeks of yoga practice as compared to baseline recording and further reduction after 6 weeks of yoga practice (Table 2). After six weeks of yoga practice all these variables were significantly reduced as compared to control group also. No such significant changes were found in the control group over the study period in within-group comparison (Table 2). In yoga group, during HGT also there was significant reduction in SBP as compared to baseline recording (Table 3), whereas, ÄSBP (change in SBP during HGT i.e. SBP during HGT minus SBP before start of HGT) increased after 4 weeks of yoga practice and further increased after 6 weeks of yoga practice (Table 3). The yoga group showed reduction in DBP also during HGT, however, it was not statistically significant. The increase in $\mathrm{ADBP}$ (change in DBP during HGT i.e. DBP during HGT minus DBP before start of HGT) was $>10 \mathrm{mmHg}$ at 0 -week recording and at subsequent 4-weeks and 6-weeks of recordings. There were no significant changes over the study period. The control group showed no significant changes in SBP or DBP during HGT in within-group comparison over the study period.

Similarly the yoga group showed significant reduction in both SBP (Fig 1 \& 2) and DBP (Fig 3) during head-up tilt test after 4 weeks of yoga practice as compared to baseline recording and further reduction after 6 weeks of yoga practice. The control group showed no significant changes in SBP or DBP during HUT in within-group comparison over the study period. The Valsalva ratio significantly increased in yoga group after 2 weeks of yoga practice (Table 2 ). Such increase was not found in control group. No significant difference was found in E:I ratio both in within- and between-group comparisons over the study period.

Dose of the anti-hypertensive drugs was reduced in four of the patients of yoga group. In one patient, dose of propranolol was reduced from $40 \mathrm{mg}$ to 20 mg after 2 weeks of yoga practice and further the dose was reduced to $10 \mathrm{mg}$ after 4 weeks of yoga practice and in another

patients dose of both propranolol $40 \mathrm{mg}$ and nifedipine $10 \mathrm{mg}$ was reduced to half after 6 weeks of yoga practice. In the similar way dose of the propranolol was reduced to half in another two patients.

Table 1. General characteristics of essential hypertensive patients

$\begin{array}{lccc}\text { Parameters } & \begin{array}{c}\text { Control }(\mathbf{n}=7) \\ (\mathbf{m e a n} \pm \mathbf{S D})\end{array} & \begin{array}{c}\text { Yoga }(\mathbf{n}=7) \\ (\mathbf{m e a n} \pm \mathbf{S D})\end{array} & \text { p value } \\ \text { Age }(\text { years }) & 42.20 \pm 11.88 & 44.86 \pm 10.76 & \mathrm{NS} \\ \text { Height }(\mathrm{cm}) & 162.4 \pm 6.95 & 160.9 \pm 9.79 & \mathrm{NS} \\ \text { Weight }(\mathrm{kg}) & 64.80 \pm 4.87 & 63.14 \pm 7.63 & \mathrm{NS} \\ \text { BMI }\left(\mathrm{kg} / \mathrm{m}^{2}\right) & 24.86 \pm 2.73 & 24.63 \pm 3.09 & \mathrm{NS} \\ \text { SBP }(\mathrm{mmHg}) & 152.86 \pm 11.13 & 152.5 \pm 13.89 & \mathrm{NS} \\ \text { DBP }(\mathrm{mmHg}) & 102.14 \pm 3.93 & 102.0 \pm 10.85 & \mathrm{NS} \\ \text { HR } \text { (beats/min) } & 75.2 \pm 10.3 & 84.57 \pm 18.43 & \mathrm{NS} \\ \text { RR }(\text { breath/min) } & 16 \pm 2.1 & 15 \pm 3.6 & \mathrm{NS}\end{array}$

$\mathrm{BMI}=$ body mass index, $\mathrm{SBP}=$ systolic blood pressure, $\mathrm{DBP}=$ diastolic blood pressure, $\mathrm{HR}=$ heart rate, $\mathrm{RR}=$ respiratory rate, $\mathrm{p}<0.05$, considered as statistical significance. $\mathrm{NS}=$ no significant differences. 
Table 2. Comparison of resting variables and over the study period, $n=7$ in each group Valsalva ratio between yoga and control groups

Variables Groups 0 -week values 2-week values 4-week values 6-week values

\begin{tabular}{|c|c|c|c|c|c|c|c|c|c|c|}
\hline \multicolumn{6}{|c|}{ Median (inter quartile range) } & \multirow{2}{*}{$\begin{array}{l}\mathrm{p} 1 \\
\mathrm{NS}\end{array}$} & \multirow{2}{*}{$\begin{array}{l}\mathrm{p} 2 \\
\mathrm{NS}\end{array}$} & \multirow{2}{*}{$\begin{array}{l}\text { p3 } \\
\text { NS }\end{array}$} & \multirow{2}{*}{$\begin{array}{l}\mathrm{p} 4 \\
\mathrm{NS}\end{array}$} & \multirow{2}{*}{$\begin{array}{l}\text { p5 } \\
\text { NS }\end{array}$} \\
\hline Weight & Control & $64(63-69)$ & $64(63-68)$ & $64(64-68)$ & $64(63-68)$ & & & & & \\
\hline kg & Yoga & $65(56.5-68)$ & $63(56-67)$ & $63(56-66.5)^{*}$ & $60(55.5-65.5)^{*}$ & NS & 0.026 & 0.027 & NS & 0.041 \\
\hline BMI, & Control & $24.7(24.2-26.5)$ & $24.4(24.2-26.5)$ & $24.7(24.2-26.5)$ & 24.6(24.4-26.9) & NS & NS & NS & NS & NS \\
\hline $\mathrm{kg} / \mathrm{m}^{2}$ & Yoga & $24.8(22.6-26)$ & $24.69(22.3-25.5)$ & $24.5(22.3-24.7)$ & $23.7(22.1-24.5)^{*}$ & NS & 0.028 & 0.028 & NS & 0.043 \\
\hline HR, & Control & 75(74-76) & $69(68-73)$ & 73(72-75) & 74(70-91) & NS & NS & NS & NS & NS \\
\hline bpm & Yoga & $84(78-94.5)$ & $76(74.5-87)$ & $72(67.5-77)$ & $72(66-74.5)^{*}$ & NS & 0.028 & 0.028 & NS & 0.034 \\
\hline SBP, & Control & $140(120-146)$ & $130(130-140)$ & $132(124-142)$ & $128(126-144)$ & NS & NS & NS & NS & NS \\
\hline mmHg & Yoga & $143(136-155)$ & $120(120-138)$ & $126(122.5-135)^{*}$ & $122(117-129)^{*}$ & NS & 0.042 & 0.018 & NS & NS \\
\hline DBP, & Control & $86(80-90)$ & 90(90-92) & 88(88-96) & 86(86-92) & NS & NS & NS & NS & NS \\
\hline mmHg & Yoga & $98(94-101)^{*}$ & $90(86-94)$ & $88(78-90)$ & $80(78-85)^{*}$ & 0.027 & 0.018 & 0.018 & 0.017 & 0.028 \\
\hline Valsalva & Control & $1.06(1.06-1.09)$ & $1.2(1.06-1.21)$ & $1.38(1.25-1.5)$ & $1.26(1.16-1.5)$ & NS & NS & NS & NS & NS \\
\hline ratio & Yoga & $1.17(1.13-1.4)$ & $1.27(1.21-1.72)$ & $1.33(1.29-1.55)$ & $1.46(1.38-2.25)^{*}$ & 0.032 & 0.018 & 0.018 & NS & 0.018 \\
\hline
\end{tabular}

Table 3. Comparison of SBP (mmHg) responses in HGT between yoga and control groups over the study period, $n=7$ in each group

\begin{tabular}{|c|c|c|c|c|c|c|c|c|c|c|}
\hline HGT & Groups & 0-week values & 2-week values & 4-week values & 6-week values & \multicolumn{5}{|c|}{ In intra-group comparisons } \\
\hline Variables & & & \multicolumn{2}{|c|}{ Median (inter quartile range) } & & p1 & p2 & p3 & $\mathrm{p} 4$ & p5 \\
\hline Baseline & Control & $132(130-140)$ & $140(140-142)$ & 136(132-140) & 134(132-138) & NS & NS & NS & NS & NS \\
\hline SBP & Yoga & $144(140-165)$ & $140(131-145)$ & $130(125-144)$ & $120(118-145)$ & 0.042 & 0.018 & 0.039 & NS & 0.039 \\
\hline SBP at & Control & $150(144-154)$ & $160(158-162)$ & $150(132-$ & 146(138-160) & NS & NS & NS & NS & NS \\
\hline 1 min & Yoga & $170(160-175)$ & $170(1$ & 160 & 148( & NS & NS & NS & NS & 0.042 \\
\hline SBP at & Control & $156(156-1$ & 166( & 160 & 160 & NS & NS & NS & NS & NS \\
\hline $2 \min$ & Yoga & 188(176-199) & $168(157-$ & $164(149-166.5)$ & $150(146-161)^{*}$ & NS & 0.018 & 0.018 & NS & 0.018 \\
\hline SBP at & Control & $170(160-172)$ & 170(168-194) & $162(160-170)$ & 172(148-180) & NS & NS & NS & NS & NS \\
\hline $4 \mathrm{~min}$ & Yoga & $188(164-198)$ & $178(165-183)$ & $170(160-182)$ & $164(160-174)$ & NS & NS & NS & NS & NS \\
\hline ÄSBP & Control & $30(30-42)$ & $38(30-44)$ & $26(20-38)$ & $38(24-40)$ & NS & NS & NS & NS & NS \\
\hline & Yoga & $20(18-28)$ & $36(26-41.5)$ & $44(34-55)$ & $48(30-63.5)^{*}$ & NS & 0.028 & 0.032 & NS & 0.028 \\
\hline
\end{tabular}

HGT=Handgrip test, SBP=systolic blood pressure, SBP at 1', 2' \& 4'= SBP during HGT at the end of 1, 2, and 4 min respectively; $\ddot{A S B P}=$ increase in SBP during 4 min of HGT; p1=0-week vs. two-week, p2=0week vs. four-week, p3= 0-week vs. six-week, p4= two-week vs. four-week, p5=two-week vs. six-week; asterisk mark $(*)=$ significant differences, control vs. yoga group; $\mathrm{p}<0.05$, considered as statistical significance, $\mathrm{NS}=$ no statistical significant difference. 


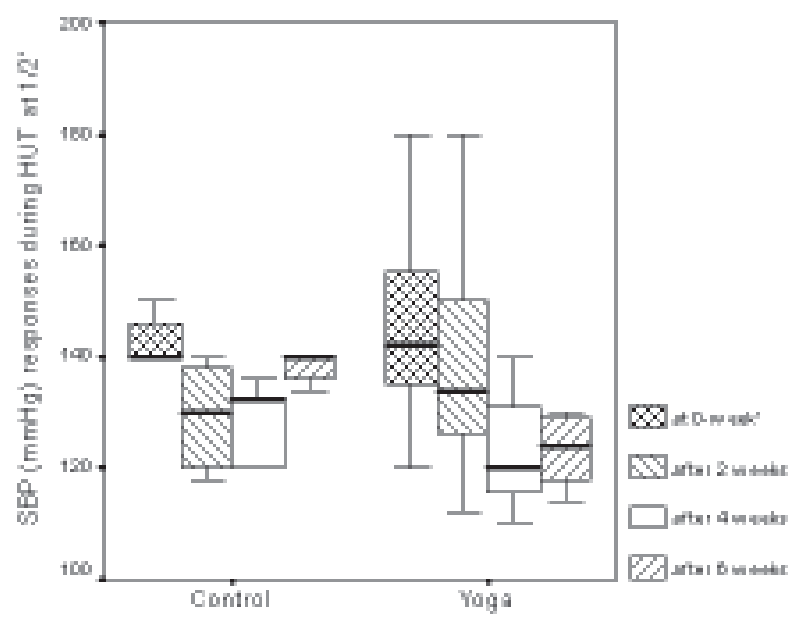

Fig 1. Changes in systolic blood pressure (SBP) responses at 1/2 min of head-up tilt test (HUT) in control $(n=7)$ and yoga $(n=7)$ groups. The yoga group showed significant reduction in SBP; 0 week vs 4-week $(p=0.027)$ and 0 -week vs 6-week $(p=0.027)$. The $p<0.05$ was considered as statistical significance.

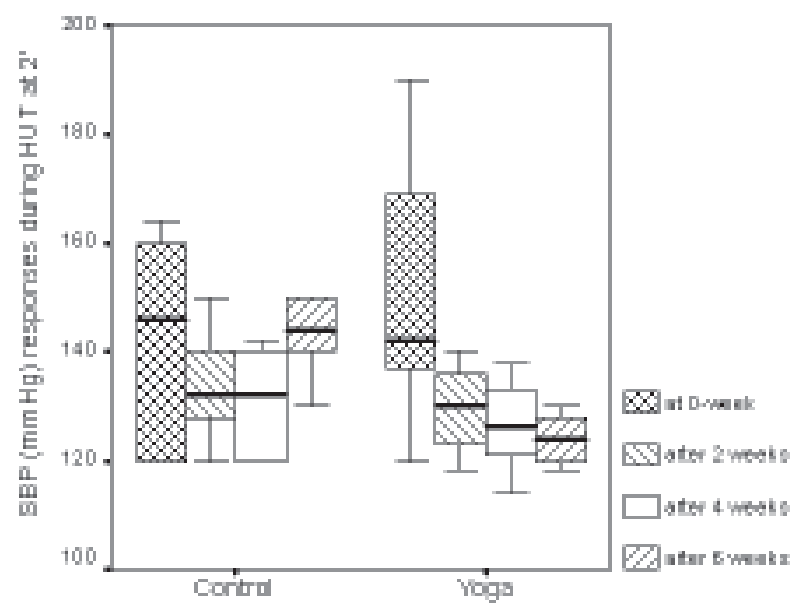

Fig 2. Changes in systolic blood pressure (SBP) responses at 2 min of head-up tilt test (HUT) in control $(n=7)$ and yoga $(n=7)$ groups. The yoga group showed significant reduction in SBP; 0 week vs 4-week $(p=0.042)$ and 0 -week vs 6-week $(p=0.028)$. The $p<0.05$ was considered as statistical significance.

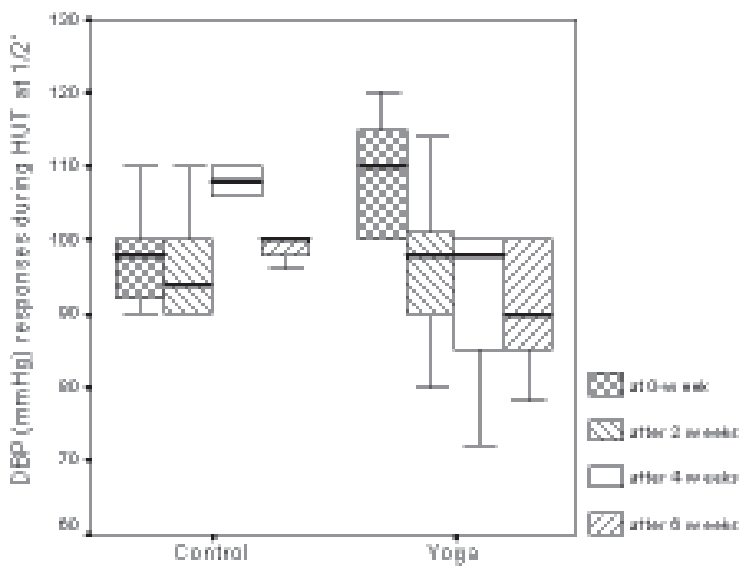

Fig 3. Changes in diastolic blood pressure (DBP) responses at 1/2 min of head-up tilt test (HUT) in control $(n=7)$ and yoga $(n=7)$ groups. The yoga group showed significant reduction in DBP; 0 week vs 4-weeks $(p=0.026)$ and 0 -week vs 6week $(p=0.018)$. The $p<0.05$ was considered as statistical significance.

\section{Discussions}

This study aimed at investigating the effect of yoga on cardiovascular autonomic reactivity in essential hypertensive patients. The study consisted of two groups; control (essential hypertensive patients on anti-hypertensive drugs) and yoga (essential hypertensive patients on similar anti-hypertensive drugs plus yogic practices). Both yoga and control groups were comparable in terms of their age, height, weight, body mass index, resting heart rate and resting blood pressure. The yoga group showed significant reduction in all these parameters (weight, BMI, HR, SBP, and DBP) after 4 weeks of yoga practice and further reduction after 6 weeks of yoga practice in within-group comparison. After six weeks of yoga practice all these variables were significantly reduced as compared to control group also, whereas, the control group showed no significant changes in weight, BMI, resting HR and resting BP within-group comparison over the study period.

In a recent study it has been reported that a 6-day yoga and diet change program decreased the BMI and the fat-free mass in healthy subjects. ${ }^{16}$ Similar result i.e. reduction in weight and BMI after yogic practices has been found in the present study also, which indicated that yoga is effective in reducing weight and BMI also in essential hypertensive patients. 
In the present study, yoga practice included combined practice of easy asanas, meditation and pranayama. The decrease in BP and HR may have been because of combined effect of components of yoga. Similar effect of decrease in SBP and DBP was reported in mild to moderate hypertensive patients. ${ }^{17,18}$ In both of the study, patients were not on antihypertensive drugs and reduction in $\mathrm{BP}$ was found after long time of yogic practices. In the present study patients were treated with antihypertensive drugs along with yoga and they showed decrease in BP even after shorter period of yoga practices.

It is important to note that drugs may have mimicry action for decreasing BP by yogic practices because similar effect in drugs treated patients was observed in an earlier study ${ }^{19}$.

Other studies ${ }^{20,21}$ also found reduction in BP after yogic practices. In these studies patients practiced meditation, pranayama, easy asanas and also few difficult asanas like Chakrasana, Halasana, and Sarbhangasana but in present study patients practiced meditation, pranayama and few easy asanas but not the difficult asanas. Thus the present study showed that combined practice of even easy asanas, meditation, and pranayama for shorter duration is effective in reducing BP in essential hypertensive patients. The duration as short as two weeks was sufficient to detect significant desirable physiological effects.

Specifically the yogic posture influences various physiological organs in the body rather than producing simple skeletal muscle action. ${ }^{22}$ A combined practice of several important asanas has shown considerable improvement in cardio-respiratory functions, adrenocortical functions and a number of metabolic correlations in addition to remarkable psychological and neurophysilogical improvements. ${ }^{23}$ It is one of the possible mechanisms for reduction of BP in hypertensive patients. The Shavasana, a relaxation exercise probably influences the hypothalamus through the continuous feedback of slow rhythmic proprioceptive and enteroreceptive impulses ${ }^{24}$ that can establish a psycho-physiological relaxation and reduce the physiological stress in shorter time..$^{25} \mathrm{It}$ is known that stress can cause hypertension through repeated blood pressure elevations as well as by stimulation of the nervous system to produce large amounts of vasoconstrictor hormones that increase blood pressure. ${ }^{26}$ Reduction in stress after yogic practices might be other possible mechanism for reduction of resting $\mathrm{HR}$ and $\mathrm{BP}$ in the present study. It is reported that yogic practices that appear to exert neuro-physiological stability is evident from lowered level of cholinesterase and catecholamines. ${ }^{27}$ It might lead to reduction in BP because lowered level of cholinesterase and catecholamines cause reduction in sympathetic activation and increase in parasympathetic activity. We also found increase in parasympathetic activity in our study after yoga practice. The Vlasalva ratio, which is a marker of parasympathetic reactivity and baroreflex function, was found to be increased after yogic practices, indicating increase in parasympathetic reactivity and baroreflex sensitivity. Another marker of parasympathetic reactivity; E:I ratio showed increasing trend. Increase in parasympathetic activity/or reactivity has obvious relation possibly reciprocally decreasing sympathetic activity. This may be one of the reasons for reduction of resting $\mathrm{HR}$ and BP in the present study.

Regarding BP changes in an earlier study it has been reported that the total peripheral resistance and average ambulatory DBP decreased significantly during meditation. ${ }^{28}$ The decrease in vasoconstrictor tone during meditation might be the hemodynamic mechanism responsible for reduction in DBP in the present study.

In the present study during handgrip test the control group showed no significant changes in SBP and DBP responses in within-group comparison over the study period, whereas the yoga group showed significant reduction in SBP during handgrip test after yogic practices. However, ÄSBP (change in SBP during HGT i.e. SBP during HGT minus SBP before start of HGT) was significantly increased after yogic practices. Similar result was obtained by Vijyalaxmi et al (2004). ${ }^{21}$ The result possibly indicates an improvement in the cardiac autonomic regulation by improving the sympathovagal balance. As a result there was reduction in SBP during rest and HGT as well and ÄSBP increased during HGT. This mechanism might make the body capable of coping up with exercise stress.

There was reduction in DBP during HGT and increase in ÄDBP over the study period, however, it was not statistical significant. The ÄDBP was $>10$ $\mathrm{mmHg}$ in baseline recording and it was maintained over the study period. The ÄDBP $>10 \mathrm{mmHg}$ is 
considered to be normal ${ }^{14}$ in healthy individuals. Thus, yoga might have not changed it because yoga is known to effect autonomic function for normalization. This fact need to be studied further.

In this study during head-up tilt both SBP and DBP were reduced after yogic practices both in supine and tilted positions and no patients showed significant postural fall during the test. In an earlier study ${ }^{29}$ progressive change in $\mathrm{BP}$ and $\mathrm{HR}$ responses to tilt during 3-week course of yogic practices was observed indicating the gradual improvement in baroreflex sensitivity. Similar mechanism might have been working in the present study.

In the present study dose of the anti-hypertensive drugs was reduced in majority of the patients of yoga group, which is supported by an earlier study. ${ }^{30}$

\section{Conclusion}

We conclude that yogic practices combined with anti-hypertensive drugs were found effective in reducing BP in resting condition and during stimulusinduced conditions as well in mild to moderate essential hypertension. It reduced the requirement of the dose of antihypertensive drugs in majority of the essential hypertensive patients. Specifically it was found to affect cardiovascular autonomic regulation and tends to normalize it. Further studies are suggested on larger sample size and with some more biochemical and stress related variables.

\section{References}

1. WHO. The World Heath Report 1998. Geneva: World Health Organization Publisher; 1998. P.88.

2. London GM, Safar ME, Safar AL, Simon AC. Blood pressure in the 'low-pressure system' and cardiac performance in essential hypertension. J Hypertens 1985; 3(4): 337-42.

3. Shigetomi S, Fukuchi S. Studies on the blood pressure control mechanism in essential hypertension: the relation of plasma norepinephrine concentrations to plasma rennin activity and haemodynamics. Nippon Naibunpi Gakkai zasshi 1980; 56(1): 78-86.

4. Esler M, Lambert G, Vaz M, Thompson J, Kaye D, Kalff V, Kelly M, Turner A, Jennings G. Central nervous system monoamine neurotransmitter turnover in primary and obesityrelated human hypertension. Clin Exp Hypertens 1997; 19(5-6):577-90.
5. Kobusiak- prokopowicz M, Negruz-Kawecka M. Heart rate variability in patients suffering from essential hypertension with different mapping of left ventricle. Pol Arch Med Wewn 2003; 109(4): 349-57.

6. Makowski K, Gielerak G, Cholewa M, Kramarz E, Michalkiewicz D, Kaminski G et al. Autonomic nervous system and left ventricular hypertrophy in essential hypertension. Kardiol Pol 2002; 57(12): 520-532.

7. Kulkarni S, O'Farrell I, Erasi M, Kochar MS. Stress and hypertension. WMJ 1998; 97(11): 34-8.

8. Kolasinska-Kloch W, Furgala A, Banach T, Laskiewicz J, Thor PJ. Circadian heart rate variability in patients with primary arterial hypertension. Przegl Lek 2002; 59(9): 752-5.

9. Mills PJ, Schneider RH, Hill D, Walton KG, Wallace RK. Beta-adrenergic receptor sensitivity in subjects practicing transcendental meditation. J Psychosom Res 1990; 34(1): 29-33.

10. Kubota Y, Sato W, Toichi M, Murai T, Okada T, Hayashi A, Sengoku A. Frontal midline theta rhythm is correlated with cardiac autonomic activities during the performance of an attention demanding meditation procedure. Brain Res Cogn Brain Res 2001; 11(2): 281-7.

11. Walton KG, Pugh ND, Gelderloos P, Macrae P. Stress reduction and preventing hypertension: preliminary support for a psychoneuroendocrine mechanism. J Altern Complement Med 1995; 1(3): 263-83.

12. Wenneberg SR, Schneider RH, Walton KG, Maclean CR, Levitsky DK, Salerno JW, et al. A controlled study of the effects of the Transcendental Meditation program on cardiovascular reactivity and ambulatory blood pressure. Int J Neurosci 1997; 89(1-2): 15-28.

13. Udupa KN, Singh RH, Settiwar RM. Physiological and Biochemical studies on the effect of yoga and certain other exercises. Indian J Med Res 1975; 63(4): 620-624.

14. Mathias CJ and Bannister R. Investigation of autonomic disorders. In: Bannister SR and Mathias CJ, editors. Autonomic Failure A Textbook of clinical disorders of the Autonomic Nervous System $3^{\text {rd }}$ ed New Work: Oxford University Press; 1992.Pp. 253-290. 
15. Ewing DJ, Clarke BF. Diagnosis and management of diabetic autonomic neuropathy. BMJ 1982; 285: 916-918.

16. Telles S, Naveen VK, Balkrishna A, Kumar S. Short term health impact of a yoga and diet change program on obesity. Med Sci Monit. 2010;16(1):CR35-40.

17. Murugesan R, Govindrajulu N, Bera TK Effect of selected yogic practices on the management of hypertension. Indian J Physiol Pharmacol 2000; 44(2):207-210.

18. Patel C, Marmot MG, Terry DJ. Controlled trail of biofeedback-aided behavioural methods in reducing mild hypertension. BMG 1981; 282:2005-2008.

19. Patel C. 12 month follow-up of yoga and biofeedback in the management of hypertension. Lancet 1975; 7898(2):62-64.

20. Laxmikanthan C, Alagesan R, Thankachalam S, Ramamurthi B, Elangovan D, Viswanathn TR, et al. Long term effects of yoga on hypertension and/or coronary disease. Jr Asso Indian 1979; 27: 1055-58.

21. Vijayalakshmi P, Madanmohan, Bhavanani AB, Patil A, Babu K. Modulation of stress induced by isometric handgrip test in hypertensive patients following yogic relaxation training. Indian J Phusiol pharmacol. 2004; 48(1): 59-64.

22. Udupa KN, Singh RH, Settuwar RM. Studies on physiological, endocrine and metabolic response to the practice of yoga in young normal volunteers. Journal Res Indian Med 1971; 6(3): 344-353.
23. Udupa KN, Singh RH. Scientific basis of yoga.Jama 1972; 220:1365.

24. Datey KK, Desmukh SN, Dalvi CP, Vixekar SL. Shavasana: A yogic exercise in the management of hypertension. Angiology 1969; 20: 325-333.

25. Bera TK, Gore MM, Oak JP. Recovey from stress in two different postures and in Shavasana -A yogic relaxation posture. Indian J Physiol Pharmacol 1998; 42(4): 473-77.

26. Kulkarni S, O’Earrell I, Erasi M, Kochar MS. Stress and hypertension. WMJ 1998; 97(11): 34-8.

27. Udupa KN, Singh RH, Settiwar RM. Physiological and Biochemical studies on the effect of yoga and certain other exercises. Indian J Med Res 1975; 63(4): 620-624.

28. Barnes VA, Treiber FA, Turner JR, Davis H, Strong WB. Acute effects of transcendental meditation on hemodynamic functioning in middle-aged adults. Psychosom Med 1999; 61(4): 525-31.

29. Silvamurthy et al, 1998 Selvamurthy W, Sridharan K, Ray US, Tiwari RS, Hegde KS, Radhakrisnan $\mathrm{U}$ et al. A physiological approach to control essential hypertension. Indian J Physiol Pharmacol 1998; 42(2): 205-13.

30. Sunder S, Agrawal SK, Singh VP, Bhattacharya SK, Udupa KN, Vaish SK. Role of yoga in management of essential hypertension. Acta Cardiol 1984; 39(3):203-8. 